Southern Illinois University Edwardsville

SPARK

SIUE Faculty Research, Scholarship, and Creative Activity

$1-2018$

\title{
Strong stability of a class of difference equations of continuous time and structured singular value problem
}

Qian Ma

Nanjing University of Science and Technology, qianmashine@gmail.com

Keqin $\mathrm{Gu}$

Southern Illinois University Edwardsville, kgu@siue.edu

Narges Choubedar

Southern Illinois University Edwardsville, nchoubedar@yahoo.co.uk

Follow this and additional works at: http://spark.siue.edu/siue_fac

Part of the Acoustics, Dynamics, and Controls Commons, Controls and Control Theory Commons, Control Theory Commons, Dynamic Systems Commons, Navigation, Guidance, Control and Dynamics Commons, Ordinary Differential Equations and Applied Dynamics Commons, and the Process Control and Systems Commons

\section{Recommended Citation}

$\mathrm{Ma}, \mathrm{Q} . \mathrm{Gu}, \mathrm{K}$. and Choubedar, N. (2018), "Strong stability of a class of difference equations of continuous time and structured singular value problem," Automatica, Vol 87, No 1, pp. 32 - 39.

This Article is brought to you for free and open access by SPARK. It has been accepted for inclusion in SIUE Faculty Research, Scholarship, and Creative Activity by an authorized administrator of SPARK. For more information, please contact magrase@siue.edu. 


\section{Cover Page Footnote}

This is an extended version of the paper published in the journal. The part of proof in the journal paper was omitted and referred to the conference version due to page limitation. This is included in this verison 


\title{
Strong stability of a class of difference equations of continuous time and structured singular value problem *
}

\author{
Qian $\mathrm{Ma}^{\mathrm{a}}$, Keqin $\mathrm{Gu}^{\mathrm{b}}$, Narges Choubedar ${ }^{\mathrm{b}}$ \\ ${ }^{a}$ School of Automation, Nanjing University of Science and Technology, Nanjing, Jiangsu 210094, China \\ ${ }^{\mathrm{b}}$ Department of Mechanical and Industrial Engineering, Southern Illinois University Edwardsville, Edwardsville, IL 62025, \\ $U S A$
}

\begin{abstract}
This article studies the strong stability of scalar difference equations of continuous time in which the delays are sums of a number of independent parameters $\tau_{i}, i=1,2, \ldots, K$. The characteristic quasipolynomial of such an equation is a multilinear function of $e^{-\tau_{i} s}$. It is known that the characteristic quasipolynomial of any difference equation set in the form of one-delayper-scalar-channel (ODPSC) model is also in such a multilinear form. However, it is shown in this article that some multilinear forms of quasipolynomials are not characteristic quasipolynomials of any ODPSC difference equation set. The equivalence between local strong stability, the exponential stability of a fixed set of rationally independent delays, and the stability for all positive delays is shown, and relations with the structured singular value problem are presented. A procedure to determine strong stability in the special case of up to three independent delay parameters in finite steps is developed. This procedure means that the structured singular value problem in the case of up to three scalar complex uncertain blocks can be solved in finite steps.
\end{abstract}

Key words: Stability; Time delay; Difference equation; Structured singular value.

\section{Introduction}

This article studies the stability problem of systems with characteristic quasipolynomial,

$$
\begin{aligned}
& \Delta(s)=1+ \\
& \sum_{m=1}^{K} \sum_{1 \leq i_{1}<i_{2}<\cdots<i_{m} \leq K} a_{i_{1} i_{2} \ldots i_{m}} e^{-\left(\tau_{i_{1}}+\tau_{i_{2}}+\cdots+\tau_{i_{m}}\right) s},
\end{aligned}
$$

where $\tau_{i}, i=1,2, \ldots, K$ are independent parameters, and $a_{i_{1} i_{2} \ldots i_{m}}$ are real coefficients. For $K=1,2$ and 3 ,

\footnotetext{
* This work is partially supported by National Science Foundation of China under Grant 61403199, the Natural Science Foundation of Jiangsu Province under Grant BK20140770, and the Fundamental Research Funds for the Central Universities of China under Grant 30916015105.

Email addresses: qianmashine@gmail.com (Qian Ma), kgu@siue.edu (Keqin Gu), nchoube@siue.edu (Narges Choubedar).
}

$\Delta(s)$ in $(1)$ becomes

$$
\begin{aligned}
\Delta(s)= & 1+a_{1} e^{-\tau_{1} s} \\
\Delta(s)= & 1+a_{1} e^{-\tau_{1} s}+a_{2} e^{-\tau_{2} s}+a_{12} e^{-\left(\tau_{1}+\tau_{2}\right) s} \\
\Delta(s)= & 1+a_{1} e^{-\tau_{1} s}+a_{2} e^{-\tau_{2} s}+a_{3} e^{-\tau_{3} s} \\
& +a_{12} e^{-\left(\tau_{1}+\tau_{2}\right) s}+a_{13} e^{-\left(\tau_{1}+\tau_{3}\right) s} \\
& +a_{23} e^{-\left(\tau_{2}+\tau_{3}\right) s}+a_{123} e^{-\left(\tau_{1}+\tau_{2}+\tau_{3}\right) s}
\end{aligned}
$$

respectively. Obviously, $\Delta(s)$ in (1) is the characteristic quasipolynomial of the difference equation of continuous time,

$$
\begin{aligned}
& y(t)+ \\
& \sum_{m=1}^{K} \sum_{1 \leq i_{1}<i_{2}<\cdots<i_{m} \leq K} a_{i_{1} i_{2} \ldots i_{m}} y\left(t-\tau_{i_{1}}-\tau_{i_{2}}-\cdots-\tau_{i_{m}}\right) \\
& =0 .
\end{aligned}
$$

As $\Delta(s)$ in (1) is a multilinear function of $e^{-\tau_{i} s}, i=$ $1,2, \ldots, K$, it is closely related to the following form of one-delay-per-scalar-channel (ODPSC) difference equa- 
tion set,

$y_{k}(t)=\sum_{j=1}^{K} d_{k j} y_{j}\left(t-\tau_{j}\right), k=1,2, \ldots, K$

where

$y_{k}(t) \in \mathbb{R}, d_{k j} \in \mathbb{R}, k, j=1,2, \ldots, K$.

Indeed, the characteristic function of (6) is

$\Delta_{1}(s)=\operatorname{det}(I-D E)=0$,

where

$$
\begin{aligned}
& D=\left(d_{i j}\right)_{K \times K}, \\
& E=\operatorname{diag}\left(e^{-\tau_{1} s}, e^{-\tau_{2} s}, \ldots, e^{-\tau_{K} s}\right) .
\end{aligned}
$$

An expansion of the determinant shows that $\Delta_{1}(s)$ is indeed a multilinear function of $e^{-\tau_{i} s}, i=1,2, \ldots, K$ in the form of (1). [7] in Section 9.6 illustrated through an example how to rewrite the difference equation of the form (5) to the ODPSC difference equation set of the form (6) for the case of $K=2$. Unfortunately, while such rewriting is always possible for $K \leq 2$, it may not be possible in some cases with $K \geq 3$ as will be shown later in this article. Therefore, studying (1) indeed has independent interest.

Difference equation of continuous time, in addition to its independent interest, also plays an important role in the theory of time-delay systems of neutral type $[5,7]$. Especially, a necessary condition for the exponential stability of the coupled differential-difference equation (8)(9) below is the exponential stability of the associated difference equation (6).

$$
\begin{aligned}
\dot{x}(t) & =A x(t)+\sum_{j=1}^{K} B_{j} y_{j}\left(t-\tau_{j}\right) \\
y_{k}(t) & =C_{k} x(t)+\sum_{j=1}^{K} d_{k j} y_{j}\left(t-\tau_{j}\right), k=1,2, \ldots, K
\end{aligned}
$$

where

$x(t) \in \mathbb{R}^{n}, y_{k}(t) \in \mathbb{R}$.

Similarly, the exponential stability of the difference equation (5) is a necessary condition for the exponential stability of the differential-difference equations of neutral type studied in [15] for $K=2$ and [6] for $K=3$. Time-delay systems of neutral type may arise in natural systems [7], or as a result of feedback control such as Smith predictor [17] and discrete implementation of distributed-delay feedback control [12-14,20].
The stability of difference equations of continuous time has been studied using the Lyapunov functional approach $[18,19]$ and frequency domain approach $[1,8,9]$. This article uses the frequency domain approach. Similar to systems described by differential equations, a system described by difference equation (5) is exponentially stable if and only if all its characteristic roots $s_{k}, k=1,2, \ldots$, i.e., the solutions of the equation

$\Delta(s)=0$,

satisfy $\operatorname{Re}\left(s_{k}\right) \leq-\epsilon$ for some $\epsilon>0$.

In this article, we concentrate on the strong stability of the system (1). In other words, we are interested in the stability of (1) when the delay parameters $\tau_{1}, \tau_{2}, \ldots, \tau_{K}$ are subject to independent, although arbitrarily small, deviation from the nominal values. The surprisingly significant impact of such small deviation was first documented by [9] and [11]. Our results are analogous to the one given by [7] and [8]. For systems with up to three independent delays, a procedure is derived that can check strong stability in finite steps.

As shown in [5], the strong stability problem of such difference equation is closely related to the structured singular value problem $[3,4,16,21]$. Therefore, the procedure derived here means that we have obtained a method to calculate the structured singular value for up to three scalar complex uncertain blocks.

The remaining parts of this article is organized as follows. Section 2 discusses the relationship between the systems described by (1) and the ODPSC model described by (7). Section 3 develops the general theory of strong stability of system (1). These two sections are very similar to the contents of [10]. Section 4 presents a method to check strong stability of the system (1) in finite steps when there are not more than three independent parameters. Section 5 discusses the relationship between the strong stability problem and the structured singular value problem. Section 6 provides some numerical examples to illustrate the developed method.

\section{Relations with ODPSC model}

From the discussion above, we know that the characteristic quasipolynominal of the ODPSC form of difference equation set (6) has the form of (1). However, as will be shown in Theorem 1 below, for a given quasipolynominal $\Delta(s)$ of the form (1) with $K \geq 3$, it is not always possible to find an ODPSC difference equation set (6) such that its characteristic function $\Delta_{1}(s)$ is equal to $\Delta(s)$. Therefore, it is of independent interest to study the system (1).

Theorem 1. For a given quasipolynominal $\Delta(s)$ in the form of (1) with $K=3$, there exists a $3 \times 3$ matrix $D$ 
such that $\Delta_{1}(s)$ given in (7) satisfies $\Delta_{1}(s)=\Delta(s)$ if and only if the following inequality holds:

$$
\begin{aligned}
& \left(a_{12} a_{3}+a_{13} a_{2}+a_{23} a_{1}-2 a_{1} a_{2} a_{3}-a_{123}\right)^{2} \\
\geq & 4\left(a_{1} a_{2}-a_{12}\right)\left(a_{2} a_{3}-a_{23}\right)\left(a_{3} a_{1}-a_{13}\right) .
\end{aligned}
$$

Proof. For the sake of convenience, write $\delta_{k}=$ $e^{-\tau_{k} s}, k=1,2,3$. Expand the determinant in (7) and simplify, we obtain

$$
\begin{aligned}
\Delta_{1}(s)= & 1-d_{11} \delta_{1}-d_{22} \delta_{2}-d_{33} \delta_{3} \\
& +D_{12} \delta_{1} \delta_{2}+D_{23} \delta_{2} \delta_{3}+D_{31} \delta_{3} \delta_{1} \\
& -\operatorname{det}(D) \delta_{1} \delta_{2} \delta_{3}
\end{aligned}
$$

where

$D_{i j}=\left|\begin{array}{ll}d_{i i} & d_{i j} \\ d_{j i} & d_{j j}\end{array}\right|$.

By matching the coefficients, it is not difficult to show that

$\Delta_{1}(s)=\Delta(s)$,

if and only if the following four equations are satisfied

$$
\begin{aligned}
& d_{12} d_{21}=a_{1} a_{2}-a_{12}, \\
& d_{23} d_{32}=a_{2} a_{3}-a_{23}, \\
& d_{13} d_{31}=a_{1} a_{3}-a_{13}, \\
& d_{21} d_{32} d_{13}+d_{31} d_{12} d_{23} \\
& =a_{12} a_{3}+a_{13} a_{2}+a_{1} a_{23}-a_{123}-2 a_{1} a_{2} a_{3} .
\end{aligned}
$$

First consider the case

$\left(a_{1} a_{2}-a_{12}\right)\left(a_{2} a_{3}-a_{23}\right)\left(a_{3} a_{1}-a_{13}\right)=0$.

Then, (11) is satisfied. Equation (18) means that at least one of the following three equations is satisfied

$a_{1} a_{2}-a_{12}=0$
$a_{2} a_{3}-a_{23}=0$,
$a_{3} a_{1}-a_{13}=0$.

Without loss of generality, suppose (19) is satisfied. Choose $d_{12}=0$. Then it is always possible to choose $d_{23}, d_{32}, d_{13}, d_{31}$ such that $d_{32} \neq 0, d_{13} \neq 0$ and (15)-(16) are satisfied. We may then choose

$d_{21}=\frac{1}{d_{32} d_{13}}\left(a_{12} a_{3}+a_{13} a_{2}+a_{1} a_{23}-a_{123}-2 a_{1} a_{2} a_{3}\right)$,

and (14)-(17) are all satisfied.

Now consider the case (18) is not satisfied. Let

$d_{12}=\alpha, d_{23}=\beta$.
Without loss of generality, we restrict $\alpha \neq 0, \beta \neq 0$. Then (14) and (15) become

$d_{21}=\frac{a_{1} a_{2}-a_{12}}{\alpha}, d_{32}=\frac{a_{2} a_{3}-a_{23}}{\beta}$,

and equation (17) can be written as

$$
\begin{aligned}
& \frac{\left(a_{1} a_{2}-a_{12}\right)\left(a_{2} a_{3}-a_{23}\right)}{\alpha \beta} d_{13}+\alpha \beta d_{31} \\
= & a_{12} a_{3}+a_{13} a_{2}+a_{1} a_{23}-a_{123}-2 a_{1} a_{2} a_{3} .
\end{aligned}
$$

Therefore, there exists a matrix $D$ such that (14)-(17) are satisfied if and only if there exist $\alpha, \beta, d_{13}$ and $d_{31}$ such that (16) and (22) are satisfied. For any given $\alpha$ and $\beta$, in the $d_{13}-d_{31}$ parameter space, the equation (16) represents a hyperbola, and (22) represents a straight line. The existence of their solutions is equivalent to the existence of intersections between the straight line and the hyperbola. We separate them into the following four cases.

\section{Case 1:}

$$
\begin{aligned}
& a_{1} a_{3}-a_{13}>0, \\
& \left(a_{1} a_{2}-a_{12}\right)\left(a_{2} a_{3}-a_{23}\right)<0 .
\end{aligned}
$$

Obviously, (11) is satisfied. In this case, the hyperbola represented by (16) is located at the first and third quadrant, and the straight line has a positive slope. The situation is illustrated in Fig. 1. It can be easily seen that they always intersect at two points.

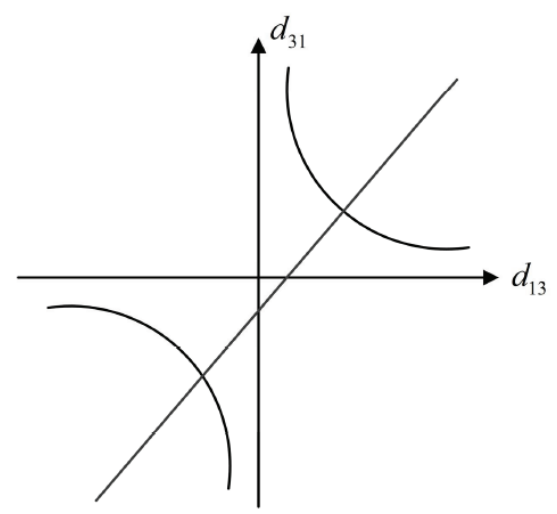

Figure 1. The location of curves represented by (16) and (22) in case 1.

Case 2:

$$
\begin{aligned}
& a_{1} a_{3}-a_{13}<0 \\
& \left(a_{1} a_{2}-a_{12}\right)\left(a_{2} a_{3}-a_{23}\right)>0 .
\end{aligned}
$$

Obviously, (11) is satisfied, the hyperbola is located at the second and fourth quadrant, and the straight line 


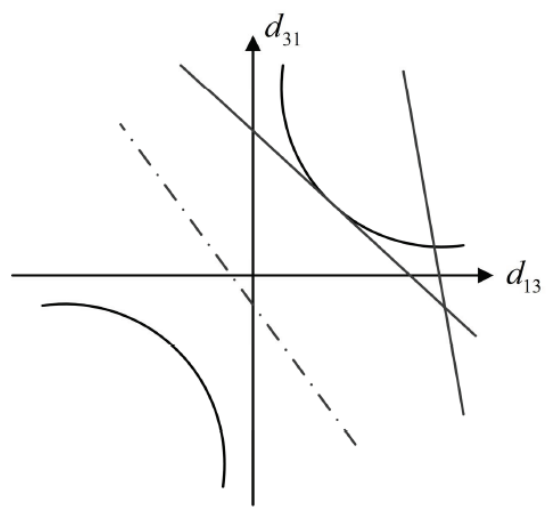

Figure 2. The location of curves represented by (16) and (22) in case 3 .

has a negative slope. Therefore, there are always two intersecting points.

Case 3:

$$
\begin{aligned}
& a_{1} a_{3}-a_{13}>0, \\
& \left(a_{1} a_{2}-a_{12}\right)\left(a_{2} a_{3}-a_{23}\right)>0 .
\end{aligned}
$$

The possibility of intersection in this case depends on the parameters. The tangent of the hyperbola at $\left(d_{130}, d_{310}\right)$ is

$d_{31}=-\left(\frac{d_{310}}{d_{130}}\right)\left(d_{13}-d_{130}\right)+d_{310}$

As $\left(d_{130}, d_{310}\right)$ needs to satisfy (16) in order to be on the hyperbola, (23) can be written as

$\frac{d_{31}}{2 d_{310}}+\frac{d_{13}}{2 \frac{a_{1} a_{3}-a_{13}}{d_{310}}}=1$.

On the other hand, the straight line described by (22) can be written as

$$
\begin{aligned}
& \frac{d_{31}}{\frac{a_{12} a_{3}+a_{13} a_{2}+a_{23} a_{1}-2 a_{1} a_{2} a_{3}-a_{123}}{\alpha \beta}} \\
& +\frac{d_{13}}{\frac{\alpha \beta\left(a_{12} a_{3}+a_{13} a_{2}+a_{23} a_{1}-2 a_{1} a_{2} a_{3}-a_{123}\right)}{\left(a_{1} a_{2}-a_{12}\right)\left(a_{2} a_{3}-a_{23}\right)}}=1 .
\end{aligned}
$$

Intersection occurs if and only if the straight line described by (25) is farther away from the origin than the tangent described by (24) when they are parallel to each other, i.e.

$$
\begin{aligned}
& \frac{\left(a_{12} a_{3}+a_{13} a_{2}+a_{23} a_{1}-2 a_{1} a_{2} a_{3}-a_{123}\right)^{2}}{\left(a_{1} a_{2}-a_{12}\right)\left(a_{2} a_{3}-a_{23}\right)} \\
\geq & 4\left(a_{1} a_{3}-a_{13}\right),
\end{aligned}
$$

which is equivalent to (11)
Case 4:

$$
\begin{aligned}
& a_{1} a_{3}-a_{13}<0, \\
& \left(a_{1} a_{2}-a_{12}\right)\left(a_{2} a_{3}-a_{23}\right)<0 .
\end{aligned}
$$

Similar to Case 3, we may conclude that the hyperbola and the straight line intersect if and only if (11) is satisfied.

All the possible cases have been exhausted, and the proof is thus complete.

\section{Stability conditions}

The strong stability condition of (6) can be found in [7] and [8] with appropriate adaption described in [5]. Here we will study the strong stability of the system (1). For complex numbers $\delta_{j}, j=1, \ldots, K$, we allow a slight abuse of notation and write

$$
\begin{aligned}
& \Delta\left(\delta_{1}, \ldots, \delta_{K}\right) \\
= & 1+\sum_{m=1}^{K} \sum_{1 \leq i_{1}<i_{2}<\cdots<i_{m} \leq K} a_{i_{1} i_{2} \ldots i_{m}} \delta_{i_{1}} \delta_{i_{2}} \ldots \delta_{i_{m}} .
\end{aligned}
$$

Then

$\Delta\left(e^{-\tau_{1} s}, e^{-\tau_{2} s}, \ldots, e^{-\tau_{K} s}\right)=\Delta(s)$

Theorem 2. The following statements are equivalent:

(i) System (1) is exponentially stable for a given set of rationally independent parameters $\tau_{1}>0, \tau_{2}>$ $0, \ldots, \tau_{K}>0$.

(ii) For given nominal parameters $\tau_{1}^{0}>0, \tau_{2}^{0}>$ $0, \ldots, \tau_{K}^{0}>0$, and an arbitrarily small $\varepsilon>0$, system (1) is exponentially stable for all positive parameters $\tau_{1}, \tau_{2}, \ldots, \tau_{K}$ that satisfy

$\left|\tau_{j}-\tau_{j}^{0}\right|<\varepsilon, j=1,2, \ldots, K$.

(iii) System (1) is exponentially stable for arbitrary positive parameters $\tau_{1}>0, \tau_{2}>0, \ldots, \tau_{K}>0$.

(iv)

$0 \notin\left\{\Delta\left(\delta_{1}, \delta_{2}, \ldots, \delta_{K}\right)|| \delta_{j} \mid \leq 1, j=1,2, \ldots, K\right\}$.

$(v)$

$$
\min \left\{\Delta\left(\delta_{1}, \delta_{2}, \ldots, \delta_{K}\right)|\Delta \in \mathbb{R},| \delta_{j} \mid=1, j=1,2, \ldots, K\right\}
$$
$>0$.

Before presenting the proof, it is worthwhile to mention that the above theorem is parallel to the one for difference equation set in [7]. Obviously, Condition (iii) (global strong stability) implies Condition (ii) (local strong stability), which in turn implies $(i)$. The fact that 
they are equivalent may be surprising for those who are not familiar with the parallel results for difference equation set. From practical point of view, if the $K$ delay parameters are not structurally contained to be rationally dependent, then they should be assumed to be subject to independent variations described by $(i i)$. The above theorem indicates that the condition for guaranteed stability in this case (no matter how accurate the estimate is) is the same as that for the case of not knowing anything about these parameters at all (other than being positive)! In view of the equivalence, we say a system is strongly stable if it satisfies any one of the above five conditions. Conditions $(i v)$ and $(v)$ are instrumental for us to check strong stability, analytically or numerically.

Proof of Theorem 2. We will show $(i v) \Leftrightarrow(v)$, $(i) \Rightarrow(v),(i v) \Rightarrow(i i i)$, and $(i i i) \Rightarrow(i i) \Rightarrow(i)$, from which the equivalence can be concluded.

$(i v) \Leftrightarrow(v)$. Define

$$
\begin{aligned}
& \Upsilon\left(\epsilon_{1}, \epsilon_{2}, \ldots, \epsilon_{K}\right) \\
= & \min \left\{\Delta\left(\delta_{1}, \delta_{2}, \ldots, \delta_{K}\right)|\Delta \in \mathbb{R},| \delta_{j} \mid \leq \epsilon_{j}, j=1,2, \ldots K\right\} .
\end{aligned}
$$

Obviously, $\Upsilon\left(\epsilon_{1}, \epsilon_{2}, \ldots, \epsilon_{K}\right)$ is a continuous and decreasing function of $\epsilon_{1}, \epsilon_{2}, \ldots, \epsilon_{K}$, and $\Upsilon(0,0, \ldots, 0)=1$. Therefore, (27) is equivalent to

$\Upsilon(1,1, \ldots, 1)>0$

For fixed $\delta_{j}=\delta_{j}^{*}, j=2,3, \ldots, K$,

$\Delta\left(\delta_{1}, \delta_{2}^{*}, \ldots, \delta_{K}^{*}\right)=b_{0}+b_{1} \delta_{1}$

where

$$
\begin{aligned}
& b_{0}=1+\sum_{m=1}^{K-1} \sum_{2 \leq i_{1}<i_{2}<\cdots<i_{m} \leq K} a_{i_{1} i_{2} \ldots i_{m}} \delta_{i_{1}}^{*} \delta_{i_{2}}^{*} \ldots \delta_{i_{m}}^{*}, \\
& b_{1}=\sum_{m=1}^{K-1} \sum_{2 \leq i_{1}<i_{2}<\cdots<i_{m} \leq K} a_{1 i_{1} i_{2} \ldots i_{m}} \delta_{i_{1}}^{*} \delta_{i_{2}}^{*} \ldots \delta_{i_{m}}^{*} .
\end{aligned}
$$

As $\delta_{1}$ varies along the unit circle $\left|\delta_{1}\right|=1, b_{0}+b_{1} \delta_{1}$ will also traces out a circle centered at $b_{0}$ with radius $\left|b_{1}\right|$ as illustrated in Fig. 3. As $\delta_{1}$ stays within the unit circle $\left|\delta_{1}\right|<1, b_{0}+b_{1} \delta_{1}$ stays within the circle shown in Fig. 3. Then, it is obvious from Fig. 3 that

$$
\min \left\{\Delta\left(\delta_{1}, \delta_{2}^{*}, \ldots, \delta_{K}^{*}\right)|\Delta \in \mathbb{R},| \delta_{1} \mid \leq 1\right\}
$$

is reached by some $\delta_{1}$ with $\left|\delta_{1}\right|=1$. Therefore,

$$
\begin{aligned}
& \min \left\{\Delta\left(\delta_{1}, \delta_{2}^{*}, \ldots, \delta_{K}^{*}\right)|\Delta \in \mathbb{R},| \delta_{1} \mid \leq 1\right\} \\
= & \min \left\{\Delta\left(\delta_{1}, \delta_{2}^{*}, \ldots, \delta_{K}^{*}\right)|\Delta \in \mathbb{R},| \delta_{1} \mid=1\right\} \\
= & \left\{\begin{array}{cl}
\operatorname{Re}\left(b_{0}\right)-\sqrt{\left|b_{1}\right|^{2}-\left[\operatorname{Im}\left(b_{0}\right)\right]^{2}}, & \left|b_{1}\right| \geq \operatorname{Im}\left(b_{0}\right) ; \\
\infty, & \left|b_{1}\right|<\operatorname{Im}\left(b_{0}\right) .
\end{array}\right.
\end{aligned}
$$

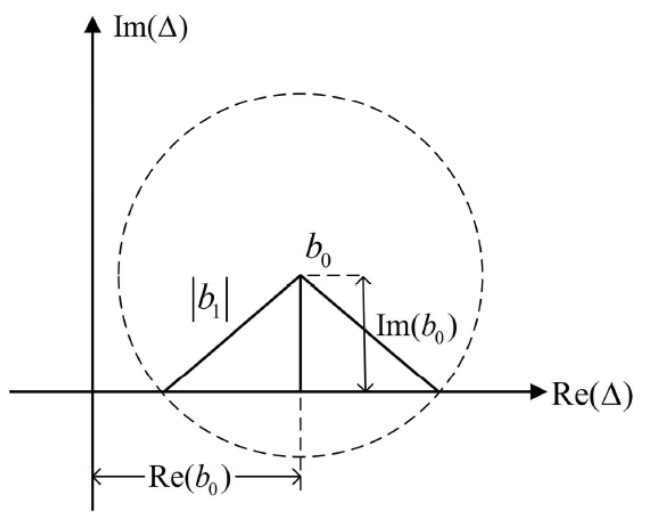

Figure 3. $\Delta\left(\delta_{1}, \delta_{2}^{*}, \ldots, \delta_{K}^{*}\right)$ as $\delta_{1}$ varies within the unit circle.

Similarly, one has

$$
\begin{aligned}
& \min \left\{\Delta\left(\delta_{1}^{*}, \delta_{2}, \delta_{3}^{*}, \ldots, \delta_{K}^{*}\right)|\Delta \in \mathbb{R},| \delta_{2} \mid=1\right\} \\
= & \min \left\{\Delta\left(\delta_{1}^{*}, \delta_{2}, \delta_{3}^{*}, \ldots, \delta_{K}^{*}\right)|\Delta \in \mathbb{R},| \delta_{2} \mid \leq 1\right\} . \\
& \ldots \ldots \\
& \min \left\{\Delta\left(\delta_{1}^{*}, \delta_{2}^{*}, \ldots, \delta_{K-1}^{*}, \delta_{K}\right)|\Delta \in \mathbb{R},| \delta_{K} \mid=1\right\} \\
= & \min \left\{\Delta\left(\delta_{1}^{*}, \delta_{2}^{*}, \ldots, \delta_{K-1}^{*}, \delta_{K}\right)|\Delta \in \mathbb{R},| \delta_{K} \mid \leq 1\right\} .
\end{aligned}
$$

Therefore, it can be concluded that the left hand side of (28) is equal to $\Upsilon(1,1, \ldots, 1)$. Hence, $(27)$ is equivalent to $(29)$, which is equivalent to (28).

$(i) \Rightarrow(v)$. Suppose $(v)$ does not hold, i.e.,

$$
\min _{\leq 0 .}\left\{\Delta\left(\delta_{1}, \ldots, \delta_{K}\right)|\Delta \in \mathbb{R},| \delta_{j} \mid=1, j=1,2, \ldots, K\right\}
$$

It is sufficient to show that $(i)$ does not hold. Define

$$
\begin{aligned}
& \varphi(\rho) \\
= & \min \left\{\Delta\left(\delta_{1}, \ldots, \delta_{K}\right)|\Delta \in \mathbb{R},| \delta_{j} \mid=e^{-\rho \tau_{j}}, j=1, \ldots, K\right\} .
\end{aligned}
$$

Then (34) means

$\varphi(0) \leq 0$

It is also obvious that

$\varphi(\infty)=1>0$

Therefore, there exists a $\rho_{0} \geq 0$ such that

$\varphi\left(\rho_{0}\right)=0$

In other words, for some $\theta_{j}^{*} \in[0,2 \pi), j=1,2, \ldots, K$,

$\delta_{j}=e^{-\left(\rho_{0} \tau_{j}+i \theta_{j}^{*}\right)}, j=1,2, \ldots, K$,

satisfy

$\Delta\left(\delta_{1}, \delta_{2}, \ldots, \delta_{K}\right)=0$. 
Since $\tau_{1}, \tau_{2}, \ldots, \tau_{K}$ are rationally independent, it follows from Kronecker theorem and basic properties of almost periodic functions [2] that, for every $\epsilon>0$, we can find a $\xi \in \mathbb{R}$ such that

$\left|\xi \tau_{j}-\theta_{j}^{*}\right|<\epsilon \bmod 2 \pi, j=1,2, \ldots, K$.

For a given series $\epsilon=\epsilon_{n} \downarrow 0$, (36) implies that there exists a corresponding sequence $\xi_{n}$ such that

$\lim _{n \rightarrow \infty} e^{i \xi_{n} \tau_{j}}=e^{i \theta_{j}^{*}}, j=1,2, \ldots, K$

Accordingly,

$\lim _{n \rightarrow \infty} \Delta\left(\delta_{1}^{(n)}, \delta_{2}^{(n)}, \ldots, \delta_{K}^{(n)}\right)=0$,

where

$\delta_{j}^{(n)}=e^{-\left(\rho_{0}+i \xi_{n}\right) \tau_{j}}, j=1,2, \ldots, K$

Because $\left|e^{i \xi_{n}}\right|=1$, and the unit circle is compact, the series $e^{i \xi_{n}}$ must have an accumulating point. Therefore, there exists a subsequence $\xi_{n_{k}}$ of $\xi_{n}$ such that $e^{i \xi_{n_{k}}} \rightarrow$ $e^{i \xi^{*}}$ as $k \rightarrow \infty$. By continuity, one has

$\Delta\left(\delta_{1}^{*}, \delta_{2}^{*}, \ldots, \delta_{K}^{*}\right)=0$

where

$\delta_{j}^{*}=e^{-\left(\rho_{0}+i \xi^{*}\right) \tau_{j}}, j=1,2, \ldots, K$.

This means that $(i)$ is violated. From this, we conclude $(i)$ implies $(v)$.

$(i v) \Rightarrow(i i i)$. Suppose that (iii) does not hold. Then, there exists at least one set of positive delays $\tau_{1}^{*}, \tau_{2}^{*}, \ldots, \tau_{K}^{*}$ such that system (1) is not exponentially stable. This means the equation

$\Delta\left(e^{-\tau_{1}^{*} s}, e^{-\tau_{2}^{*} s}, \ldots, e^{-\tau_{K}^{*} s}\right)=0$

has a series of solutions $s_{n}, n=1,2, \cdots$, such that $\lim _{n \rightarrow \infty} \operatorname{Re}\left(s_{n}\right)=\rho^{*} \geq 0$. Let $\theta_{n}=\operatorname{Im}\left(s_{n}\right)$. Because $\left|e^{i \theta_{n}}\right|=1$, and the unit circle is compact, the series $e^{i \theta_{n}}$ must have an accumulating point. Then, there exists a subsequence $\theta_{n_{k}}$ of $\theta_{n}$ such that $e^{i \theta_{n_{k}}} \rightarrow e^{i \theta^{*}}$ as $k \rightarrow \infty$. By continuity, we conclude that $\delta_{j}=e^{-\left(\rho^{*}+i \theta^{*}\right) \tau_{j}}$ satisfies (35) but $\left|\delta_{j}\right|=e^{-\rho^{*} \tau_{j}} \leq 1$. Therefore, this violates the statement $(i v)$. It can thus be concluded that $(i v) \Rightarrow($ iii $)$.

The fact that $(i i i) \Rightarrow(i i) \Rightarrow(i)$ is obvious, and thus the proof is complete.

\section{Stability conditions for $K \leq 3$}

In this section, we will express the strong stability conditions for $K \leq 3$ in a form that can be checked in finite steps. Obviously, such strong stability conditions are of interest. For example, Assumption 3 in [15] and Assumption III in [6] may be replaced by the strong stability conditions of the difference equations developed here to reduce conservatism. In addition, this also leads to a method of calculating structured singular value with no more than 3 complex scalar blocks as will be shown later. The following lemma is instrumental.

Lemma 3. For given $\delta_{2}=\delta_{2}^{*}, \delta_{3}=\delta_{3}^{*}, \ldots, \delta_{K}=\delta_{K}^{*}$,

$\min \left\{\Delta\left(\delta_{1}, \delta_{2}^{*}, \ldots, \delta_{K}^{*}\right)|\Delta \in \mathbb{R},| \delta_{1} \mid=1\right\}>0$

is satisfied if and only if either one of the following two conditions holds:

i)

ii)

$$
\begin{aligned}
& \left|\operatorname{Im}\left(b_{0}\right)\right|>\left|b_{1}\right| ; \\
& \operatorname{Re}\left(b_{0}\right)>0, \quad \text { and } \\
& \left|b_{0}\right|>\left|b_{1}\right|,
\end{aligned}
$$

where

$b_{0}=1+\sum_{m=1}^{K-1} \sum_{2 \leq i_{1}<i_{2}<\cdots<i_{m} \leq K} a_{i_{1} i_{2} \ldots i_{m}} \delta_{i_{1}}^{*} \delta_{i_{2}}^{*} \ldots \delta_{i_{m}}^{*}$

$b_{1}=\sum_{m=1}^{K-1} \sum_{2 \leq i_{1}<i_{2}<\cdots<i_{m} \leq K} a_{1 i_{1} i_{2} \ldots i_{m}} \delta_{i_{1}}^{*} \delta_{i_{2}}^{*} \ldots \delta_{i_{m}}^{*}$.

Proof. Similar to the proof of Theorem $2(i v) \Leftrightarrow(v)$, it is obvious from Fig. 3 or (31).

The following theorem for $K=3$ follows easily from the above lemma.

Theorem 4. The system (4) is strongly stable if and only if the following two conditions are both satisfied for all $\left|\delta_{2}^{*}\right|=\left|\delta_{3}^{*}\right|=1$ :

$$
\begin{aligned}
& \text { i) } \quad\left|1+a_{2} \delta_{2}^{*}+a_{3} \delta_{3}^{*}+a_{23} \delta_{2}^{*} \delta_{3}^{*}\right| \\
& >\left|a_{1}+a_{12} \delta_{2}^{*}+a_{13} \delta_{3}^{*}+a_{123} \delta_{2}^{*} \delta_{3}^{*}\right| . \\
& \text { ii) Either } \operatorname{Re}\left(1+a_{2} \delta_{2}^{*}+a_{3} \delta_{3}^{*}+a_{23} \delta_{2}^{*} \delta_{3}^{*}\right)>0 \text {, } \\
& \text { or } \quad\left|\operatorname{Im}\left(1+a_{2} \delta_{2}^{*}+a_{3} \delta_{3}^{*}+a_{23} \delta_{2}^{*} \delta_{3}^{*}\right)\right| \\
& >\left|a_{1}+a_{12} \delta_{2}^{*}+a_{13} \delta_{3}^{*}+a_{123} \delta_{2}^{*} \delta_{3}^{*}\right| \text {. }
\end{aligned}
$$

Proof. In Lemma 3, (41) implies (43). Therefore, the necessary and sufficient conditions for (40) can be equivalently stated as the following two conditions are both satisfied: i) (43) is satisfied, and ii) either (42) or (41) is satisfied. The proof is complete by recognizing that (43) becomes (44), (42) becomes (45), and (41) becomes (46) when $K=3$. 
We now present a method to check (44) as a theorem. The proof is given in the Appendix.

Theorem 5. Let

$p_{1}(z)=C_{2} z^{2}+C_{1} z+C_{1} z^{-1}+C_{2} z^{-2}+C_{0}$,

where

$C_{2}=g_{2}^{2}-4 f_{2} f_{3}$

$C_{1}=2 g_{1} g_{2}-4 f_{1}\left(f_{2}+f_{3}\right)$,

$C_{0}=g_{1}^{2}+2 g_{2}^{2}-4\left(f_{1}^{2}+f_{2}^{2}+f_{3}^{2}\right)$,

and

$f_{1}=a_{3}+a_{2} a_{23}-a_{1} a_{13}-a_{12} a_{123}$,

$f_{2}=a_{23}-a_{1} a_{123}$,

$f_{3}=a_{2} a_{3}-a_{12} a_{13}$,

$g_{1}=a_{1}^{2}+a_{13}^{2}+a_{12}^{2}+a_{123}^{2}-a_{3}^{2}-a_{2}^{2}-a_{23}^{2}-1$,

$g_{2}=a_{1} a_{12}+a_{13} a_{123}-a_{2}-a_{3} a_{23}$.

Let $z_{i}^{*},\left|z_{i}^{*}\right|=1, i=1,2, \ldots, n, n \leq 4$ denote all the solutions to the following equation on the unit circle,

$p_{2}(z)=2 C_{2} z^{4}+C_{1} z^{3}-C_{1} z-2 C_{2}=0$.

Then, the inequality (44) holds for all $\left|\delta_{2}^{*}\right|=\left|\delta_{3}^{*}\right|=1$ if and only if

$\left\{\begin{array}{l}p_{1}\left(z_{i}^{*}\right)>0, i=1,2, \ldots, n, \\ -2\left|g_{2}\right|>g_{1} .\end{array}\right.$

Next we will consider (45). For $\delta_{2}^{*}$ and $\delta_{3}^{*}$ on the unit circle, we may write

$\delta_{2}^{*}=\cos (\alpha)+i \sin (\alpha)$

$\delta_{3}^{*}=\cos (\beta)+i \sin (\beta)$,

and the left hand side of (45) becomes

$\eta(\alpha, \beta)=1+a_{2} \cos (\alpha)+a_{3} \cos (\beta)+a_{23} \cos (\alpha+\beta)$.

Checking (45) reduces to the minimization of $\eta(\alpha, \beta)$. The solution is given in the following theorem. The proof is given in the Appendix.

Theorem 6. A local minimum of $\eta(\alpha, \beta)$ may be reached by $\alpha$ and $\beta$ that satisfy

$\sin (\alpha)=0, \sin (\beta)=0$,

and the corresponding value of $\eta(\alpha, \beta)$ is either

$\eta_{r_{+}}=1+a_{2}-\left|a_{3}+a_{23}\right|$ or

$\eta_{r_{-}}=1-a_{2}-\left|a_{3}-a_{23}\right|$

Furthermore, if

$a_{2} \neq 0, a_{3} \neq 0, a_{23} \neq 0$,

and

$\left|\frac{1}{2}\left(\frac{a_{3} a_{23}}{a_{2}^{2}}-\frac{a_{3}}{a_{23}}-\frac{a_{23}}{a_{3}}\right)\right| \leq 1$,
$\left|\frac{1}{2}\left(\frac{a_{2} a_{23}}{a_{3}^{2}}-\frac{a_{2}}{a_{23}}-\frac{a_{23}}{a_{2}}\right)\right| \leq 1$,

then a local minimum may also be reached by $\alpha$ and $\beta$ that satisfy

$\cos (\alpha)=\frac{1}{2}\left(\frac{a_{3} a_{23}}{a_{2}^{2}}-\frac{a_{3}}{a_{23}}-\frac{a_{23}}{a_{3}}\right)$,

$\cos (\beta)=\frac{1}{2}\left(\frac{a_{2} a_{23}}{a_{3}^{2}}-\frac{a_{2}}{a_{23}}-\frac{a_{23}}{a_{2}}\right)$,

$\sin (\alpha)= \pm \sqrt{1-\cos ^{2} \alpha}$,

$\sin (\beta)=\frac{a_{2}}{a_{3}} \sin (\alpha)$.

The corresponding local minimum is

$\eta_{c}=1-\frac{1}{2}\left(\frac{a_{2} a_{3}}{a_{23}}+\frac{a_{2} a_{23}}{a_{3}}+\frac{a_{3} a_{23}}{a_{2}}\right)$.

The global minimum is

$\min _{0 \leq \alpha, \beta \leq 2 \pi} \eta(\alpha, \beta)=\min \left\{\eta_{r_{+}}, \eta_{r_{-}}, \eta_{c}\right\}$

if (56), (57) and (58) are all satisfied. Otherwise,

$\min _{0 \leq \alpha, \beta \leq 2 \pi} \eta(\alpha, \beta)=\min \left\{\eta_{r_{+}}, \eta_{r_{-}}\right\}$.

From the above results, we arrive at the following algorithm.

Algorithm 1. (For checking the strong stability of the system described by (4) using Theorem 4).

Step 1. Check if (44) holds for all $\delta_{2}^{*}, \delta_{3}^{*}$ on the unit circle. If not, declare the system not strongly stable, and terminate the algorithm. Otherwise, continue.

Step 2. Check if $\eta_{r_{+}}$given in (54) and $\eta_{r_{-}}$given in (55) are both positive. If either of them is not positive, declare the system not strongly stable, and terminate the algorithm. Otherwise, continue.

Step 3. Check if (56), (57) and (58) are all satisfied. If any of them is not satisfied, then declare the system strongly stable, and terminate the algorithm. Otherwise, continue. 
Step 4. Check if $\eta_{c}$ given in (63) is positive. If it is, then declare the system strongly stable, and terminate the algorithm. Otherwise, continue.

Step 5. Check if (46) is satisfied by $\delta_{2}^{*}$ and $\delta_{3}^{*}$ given in (50) and $(51)$, where $\cos (\alpha), \cos (\beta), \sin (\alpha)$ and $\sin (\beta)$ are given in (59)-(62). If not, declare that the system not strongly stable. Otherwise, declare the strong stability cannot be determined by this algorithm. Terminate the algorithm.

In Step 2, if either $\eta_{r_{+}}$or $\eta_{r_{-}}$is not positive, then (45) is violated by some real $\delta_{2}^{*}$ and $\delta_{3}^{*}$. Obviously, (46) is also violated as its left hand side vanishes. In Step 3, if (56), (57) and (58) are not all satisfied, then (65) holds. In Step 5 , if (46) is satisfied by the given $\delta_{2}^{*}$ and $\delta_{3}^{*}$, it is insufficient to determine the system to be strongly stable. Indeed, in this case, the strong stability requires (46) to be satisfied by all $\delta_{2}^{*}$ and $\delta_{3}^{*}$ in the following set

$$
\left\{\left(\delta_{2}^{*}, \delta_{3}^{*}\right)|| \delta_{2}^{*}|=1,| \delta_{3}^{*} \mid=1,(45) \text { is not satisfied }\right\} \text {, }
$$

which is not easy to determine. Fortunately, the problem can be circumvented by changing the roles of $\delta_{1}, \delta_{2}$ and $\delta_{3}$ as described below.

Theorem 4 is based on first calculating the minimum for $\left|\delta_{1}\right|=1$ with fixed $\delta_{2}^{*}$ and $\delta_{3}^{*}$. Parallel results can be obtained by first calculating the minimum for $\left|\delta_{2}\right|=1$ with fixed $\delta_{1}^{*}$ and $\delta_{3}^{*}$, or the minimum for $\left|\delta_{3}\right|=1$ with fixed $\delta_{1}^{*}$ and $\delta_{2}^{*}$. These parallel results are given below as Theorems 7 and 8 . They can be obtained from Theorem 4 by alternating the subscripts 1,2 , and 3 .

Theorem 7. The system (4) is strongly stable if and only if the following two conditions are satisfied for all $\left|\delta_{1}^{*}\right|=\left|\delta_{3}^{*}\right|=1$ :

i)

$$
\begin{gathered}
\left|1+a_{1} \delta_{1}^{*}+a_{3} \delta_{3}^{*}+a_{13} \delta_{1}^{*} \delta_{3}^{*}\right| \\
>\left|a_{2}+a_{12} \delta_{1}^{*}+a_{23} \delta_{3}^{*}+a_{123} \delta_{1}^{*} \delta_{3}^{*}\right| .
\end{gathered}
$$

ii) Either $\operatorname{Re}\left(1+a_{1} \delta_{1}^{*}+a_{3} \delta_{3}^{*}+a_{13} \delta_{1}^{*} \delta_{3}^{*}\right)>0$, or

$$
\begin{aligned}
& \left|\operatorname{Im}\left(1+a_{1} \delta_{1}^{*}+a_{3} \delta_{3}^{*}+a_{13} \delta_{1}^{*} \delta_{3}^{*}\right)\right| \\
> & \left|a_{2}+a_{12} \delta_{1}^{*}+a_{23} \delta_{3}^{*}+a_{123} \delta_{1}^{*} \delta_{3}^{*}\right| .
\end{aligned}
$$

Theorem 8. The system (4) is strongly stable if and only if the following two conditions are satisfied for all $\left|\delta_{1}^{*}\right|=\left|\delta_{2}^{*}\right|=1$ :

$$
\begin{aligned}
& \text { i) } \quad\left|1+a_{1} \delta_{1}^{*}+a_{2} \delta_{2}^{*}+a_{12} \delta_{1}^{*} \delta_{2}^{*}\right| \\
& >\left|a_{3}+a_{13} \delta_{1}^{*}+a_{23} \delta_{2}^{*}+a_{123} \delta_{1}^{*} \delta_{2}^{*}\right| . \\
& \text { ii) Either } \operatorname{Re}\left(1+a_{1} \delta_{1}^{*}+a_{2} \delta_{2}^{*}+a_{12} \delta_{1}^{*} \delta_{2}^{*}\right)>0 \text {, } \\
& \text { or } \quad\left|\operatorname{Im}\left(1+a_{1} \delta_{1}^{*}+a_{2} \delta_{2}^{*}+a_{12} \delta_{1}^{*} \delta_{2}^{*}\right)\right| \\
& >\left|a_{3}+a_{13} \delta_{1}^{*}+a_{23} \delta_{2}^{*}+a_{123} \delta_{1}^{*} \delta_{2}^{*}\right| \text {. }
\end{aligned}
$$

Satisfaction of Theorem 7 or 8 may also be checked using parallel algorithms (which will be called Algorithms 2 and 3, respectively) obtained from Algorithm 1 by alternating subscripts. It should be pointed out that although Theorems 4, 7 and 8 are equivalent, Algorithms
1,2 ,and 3 are not. As will be illustrated in Section 6 , it is sometimes necessary to apply Algorithm 2 or 3 to obtain a definite answer if Algorithm 1 fails to do so. All the systems we have tested seem to indicate that we can always obtain a definite answer after all three algorithms have been used although we have not been able to theoretically prove this.

We will turn our attention to the case of $K=2$. Obviously, this is a special case of $K=3$, and Theorem 4 and Algorithm 1 still apply. However, more explicit conditions are possible in this case as is presented below.

Theorem 9. The system (3) is strongly stable if and only if the following two inequalities hold:

$\left\{\begin{array}{l}1-a_{2}>\left|a_{1}-a_{12}\right|, \\ 1+a_{2}>\left|a_{1}+a_{12}\right|,\end{array}\right.$

or equivalently, the following two inequalities hold:

$\left\{\begin{array}{l}1-a_{1}>\left|a_{2}-a_{12}\right|, \\ 1+a_{1}>\left|a_{2}+a_{12}\right| .\end{array}\right.$

Proof. Suppose the system is strongly stable. Then Theorem 2 (iv) requires

$0 \notin\left\{\Delta\left(\delta_{1}, \delta_{2}\right)|| \delta_{1}|\leq 1,| \delta_{2} \mid \leq 1\right\}$.

The above implies

$\left|a_{1}\right|<1$

and

$\left|a_{2}\right|<1$

(76) implies that the condition (45) is true for $\delta_{3}^{*}=0$ and all $\left|\delta_{2}^{*}\right| \leq 1$. Therefore, we conclude that necessary and sufficient conditions for $\min \left\{\Delta\left(\delta_{1}, \delta_{2}\right)|\Delta \in \mathbb{R},| \delta_{1} \mid=\right.$ $\left.1,\left|\delta_{2}\right|=1\right\}>0$ are (76) and (44) hold for $\delta_{3}^{*}=0$ and all $\left|\delta_{2}^{*}\right|=1$. (44) can be rewritten as

$\left(1+a_{2} \delta_{2}^{*}\right) \overline{\left(1+a_{2} \delta_{2}^{*}\right)}>\left(a_{1}+a_{12} \delta_{2}^{*}\right) \overline{\left(a_{1}+a_{12} \delta_{2}^{*}\right)}$,

or

$a_{1}^{2}+a_{12}^{2}-1-a_{2}^{2}<2 \operatorname{Re}\left[\left(a_{2}-a_{1} a_{12}\right) \delta_{2}^{*}\right]$.

The above holds for all $\left|\delta_{2}^{*}\right|=1$ if and only if

$a_{1}^{2}+a_{12}^{2}-1-a_{2}^{2}<-2\left|a_{2}-a_{1} a_{12}\right|$.

(79) is equivalent to

$2\left(a_{2}-a_{1} a_{12}\right)<-\left(a_{1}^{2}+a_{12}^{2}-1-a_{2}^{2}\right)$, 
and

$2\left(a_{2}-a_{1} a_{12}\right)>a_{1}^{2}+a_{12}^{2}-1-a_{2}^{2}$.

It is easily seen that (80) and (81) along with (76) are equivalent to (72). Thus (72) is necessary and sufficient. Note that the first inequality in (72) is equivalent to (82) and (83) below, and the second inequality in (72) is equivalent to (84) and (85) below.

$$
\left\{\begin{array}{l}
a_{12}-a_{2}>a_{1}-1, \\
a_{2}+a_{12}<1+a_{1}, \\
a_{12}-a_{2}<1-a_{1}, \\
a_{2}+a_{12}>-1-a_{1} .
\end{array}\right.
$$

But (82) and (84) are equivalent to the first inequality of (73), and (83) and (85) are equivalent to the second inequality of (73). This shows that (72) is equivalent to (73). The proof is complete.

In Section 9.6 of [7], the same stability conditions (after correcting a sign error and adapting the notation) were obtained by rewriting it to a set of two difference equations and appealing to the Routh-Hurwitz criteria with complex coefficients. The proof here is much simpler.

The case for $K=1$ is obvious, and is stated as follows.

Theorem 10. The system (2) is strongly stable if and only if the following inequality holds:

$\left|a_{1}\right|<1$.

\section{Relations with structured singular value problem}

The strong stability problem of ODPSC difference equation set is closely related to the structured singular value problem $[3,4,16]$. Indeed, as discussed in [5], the difference equation set (6) is strongly stable if and only if

$\rho_{0} \triangleq \sup _{\substack{\delta_{k} \in \mathbb{R},\left|\delta_{k}\right|=1 \\ k=1,2, \ldots, K}} \rho(D E(\delta))<1$

where

$E(\delta)=\operatorname{diag}\left(\delta_{1}, \delta_{2}, \ldots, \delta_{K}\right)$

It is not difficult to show that $\left|\delta_{k}\right|=1$ may be relaxed to $\left|\delta_{k}\right| \leq 1$,

$\rho_{0}=\sup _{\substack{\delta_{k} \in \mathbb{R},\left|\delta_{k}\right| \leq 1 \\ k=1,2, \ldots, K}} \rho(D E(\delta))$.
In view of (89), the stability condition (87) can be equivalently expressed as a structured singular value problem

$\mu(D)<1$

where

$$
=\frac{\mu(D)}{\min \left\{r \mid \operatorname{det}(I-D E(\delta))=0, \text { for some }\left|\delta_{k}\right| \leq r, k=1, \ldots, K\right\}}
$$

is the structured singular value of the matrix $D$ under the uncertainty structure of $K$ complex scalar blocks of size one each.

For a given multilinear expression $\Delta\left(\delta_{1}, \delta_{2}, \ldots, \delta_{K}\right)$, we may also define

$$
=\frac{\mu(\Delta)}{\min \left\{r \mid \Delta\left(\delta_{1}, \ldots, \delta_{K}\right)=0 \text { for some }\left|\delta_{k}\right| \leq r, k=1, \ldots, K\right\}} .
$$

Then, it is immediately clear that $\Delta(s)$ is strongly stable if and only if

$\mu(\Delta)<1$

In view of the fact that $\Delta_{1}(s)$ is the characteristic quasipolynomial of the ODPSC difference equation set, it is easily seen that

$\mu(D)=\mu\left(\Delta_{1}\right)$

Therefore, the results presented in the last section means that we have obtained a method to check the satisfaction of $\mu(D)<1$ in finite steps for the uncertainty structure of up to three complex scalar blocks.

\section{Illustrative examples}

In this section, we present some numerical examples to illustrate the method developed in Section 4.

Example 1. Consider a system described by (4) with the following parameters

$$
\begin{aligned}
& a_{1}=0.4, a_{2}=0.3, a_{3}=0.1, a_{12}=0.15 \\
& a_{13}=-0.2, a_{23}=0.5, a_{123}=0.1
\end{aligned}
$$

Apply Algorithm 1, we find that (44) is satisfied for all $\delta_{2}^{*}$ and $\delta_{3}^{*}$ on the unit circle. $\eta_{r_{+}}$and $\eta_{r_{-}}$are both positive, but neither (57) nor (58) is satisfied. Therefore, we can conclude that the system is strongly stable, and the algorithm terminates in Step 3. 
Because (11) is not satisfied, the system cannot be written in the form of ODPSC model.

Example 2. Consider a system described by (4) with the following parameters

$$
\begin{aligned}
& a_{1}=0.3, a_{2}=0.3, a_{3}=0.8, a_{12}=-0.1, \\
& a_{13}=0.2, a_{23}=-0.2, a_{123}=-0.1 .
\end{aligned}
$$

Apply Algorithm 1, we find that (44) is satisfied for all $\delta_{2}^{*}$ and $\delta_{3}^{*}$ on the unit circle. (55) is nonpositive. Therefore, we conclude that system is not strongly stable. The algorithm terminates in Step 2.

Because (11) is satisfied, then there exists a $D$ such that $\Delta_{1}(s)=\Delta(s)$ according to Theorem 1 . Indeed, by following its proof, we may find such a $D$,

$D=\left[\begin{array}{ccc}-0.3 & 1 & -0.474 \\ 0.19 & -0.3 & 1 \\ -0.084 & 0.44 & -0.8\end{array}\right]$

We may confirm our conclusion by applying the stability condition of the ODPSC model given in [5] or check if $\mu(D)<1$ is satisfied by using the method given in [16].

Example 3. Consider a system described by (4) with the following parameters

$$
\begin{aligned}
& a_{1}=0.27, a_{2}=0.65, a_{3}=0.75, a_{12}=0.2, \\
& a_{13}=0.2, a_{23}=0.85, a_{123}=0.25
\end{aligned}
$$

Apply Algorithm 1, we find that (44) is satisfied for all $\delta_{2}^{*}$ and $\delta_{3}^{*}$ on the unit circle. We also find that $\eta_{r_{+}}>0$, $\eta_{r_{-}}>0$. Furthermore, (56), (57) and (58) are all satisfied, and $\eta_{c}<0$. The corresponding minimizing parameters satisfy

$$
\begin{aligned}
& \cos (\alpha) \approx-0.2534, \sin (\alpha) \approx \pm 0.9673 \\
& \cos (\beta) \approx-0.5450, \sin (\beta) \approx \pm 0.8383 .
\end{aligned}
$$

Moreover, (46) is satisfied by $\delta_{2}^{*}, \delta_{3}^{*}$ given in (50) and (51) with $\alpha, \beta$ specified above. Therefore, the strong stability of the system cannot be determined as Algorithm 1 terminates in Step 5.

However, a definite answer can be easily reached by using Algorithm 2 based on Theorem 7. Indeed, it can be checked that both (66) and (67) are satisfied for all $\delta_{1}^{*}$ and $\delta_{3}^{*}$ on the unit circle, from which we conclude that the system is strongly stable. As (11) is not satisfied, there is no ODPSC model with this characteristic quasipolynomial.

Example 4. Consider a system described by (4) with the following parameters

$$
\begin{aligned}
& a_{1}=0.32, a_{2}=0.7, a_{3}=0.75, a_{12}=0.32, \\
& a_{13}=0.32, a_{23}=0.8, a_{123}=0.32 .
\end{aligned}
$$

Similar to Example 2, (44) is satisfied for all $\delta_{2}^{*}$ and $\delta_{3}^{*}$ on the unit circle. The condition (45) is not satisfied by the minimizing $\delta_{2}^{*}, \delta_{3}^{*}$ given in (50) and (51) with

$$
\begin{aligned}
& \cos (\alpha) \approx-0.3898, \sin (\alpha) \approx \pm 0.9208 \\
& \cos (\beta) \approx-0.5111, \sin (\beta) \approx \pm 0.8594 .
\end{aligned}
$$

But the condition (46) is satisfied by this pair of $\delta_{2}^{*}$ and $\delta_{3}^{*}$, and Algorithm 1 terminates in Step 5 without a definite conclusion about strong stability.

Applying Algorithm 2, we find (66) is not satisfied for all $\delta_{1}^{*}$ and $\delta_{3}^{*}$ on the unit circle. Therefore, we can conclude that the system is not strongly stable.

The parameters for the system satisfy (11), and for

$D=\left[\begin{array}{ccc}-0.32 & 1 & 3.33 \\ -0.096 & -0.7 & 1 \\ -0.024 & -0.275 & -0.75\end{array}\right]$,

we have $\Delta_{1}(s)=\Delta(s)$. The conclusion about nonstrong stability can be confirmed by other methods mentioned in Example 2.

\section{Conclusions}

For scalar difference equations of continuous time with delays being the sum of a number of independent parameters, the following three conditions are equivalent: 1 . It is exponentially stable for a fixed set of rationally independent parameters; 2 . It is locally strongly stable; 3 . It is globally strongly stable. Although this conclusion is similar to the case of ODPSC case, and all ODPSC model share the same characteristic quasipolynomial with such a scalar difference equation, the reverse is not always true, and therefore, such a study is of independent interest. The conditions for strong stability is developed. Especially, for the case of three or less independent parameters, the strong stability conditions can be determined in finite steps.

The strong stability problem of a class of such systems is equivalent to the structured singular value problem. Therefore, the solution of this problem implies that we have found a method to solve the structured singular value problem in finite steps when the uncertainty structure is three or fewer complex scalar blocks.

\section{Appendix}




\section{Proof of Theorem 5. Let}

$c_{0}=1+a_{2} \delta_{2}^{*}, c_{1}=a_{3}+a_{23} \delta_{2}^{*}$,

$d_{0}=a_{1}+a_{12} \delta_{2}^{*}, d_{1}=a_{13}+a_{123} \delta_{2}^{*}$.

Then, (44) becomes

$\left|d_{0}+d_{1} \delta_{3}^{*}\right|<\left|c_{0}+c_{1} \delta_{3}^{*}\right|$

Take square on both sides of the above inequality and expand, we obtain

$\left|d_{0}\right|^{2}+\left|d_{1}\right|^{2}-\left|c_{0}\right|^{2}-\left|c_{1}\right|^{2}<2 \operatorname{Re}\left[\left(\bar{c}_{0} c_{1}-\bar{d}_{0} d_{1}\right) \delta_{3}^{*}\right]$.

This is true for all $\left|\delta_{3}^{*}\right|=1$ if and only if

$\left|d_{0}\right|^{2}+\left|d_{1}\right|^{2}-\left|c_{0}\right|^{2}-\left|c_{1}\right|^{2}<-2\left|\bar{c}_{0} c_{1}-\bar{d}_{0} d_{1}\right|$,

which can be equivalently expressed as

$\left\{\begin{array}{l}{\left[\left|d_{0}\right|^{2}+\left|d_{1}\right|^{2}-\left|c_{0}\right|^{2}-\left|c_{1}\right|^{2}\right]^{2}>4\left|\bar{c}_{0} c_{1}-\bar{d}_{0} d_{1}\right|^{2}(96)} \\ \left|d_{0}\right|^{2}+\left|d_{1}\right|^{2}-\left|c_{0}\right|^{2}-\left|c_{1}\right|^{2}<0 .\end{array}\right.$

Set $\delta_{2}^{*}=e^{i \theta}, \theta \in[0,2 \pi]$, and let

$\kappa(\theta)=C_{2} e^{2 i \theta}+C_{1} e^{i \theta}+C_{1} e^{-i \theta}+C_{2} e^{-2 i \theta}+C_{0}$,

then the first inequality of (96) can be written as

$\kappa(\theta)>0$.

Notice that $\min _{\theta \in[0,2 \pi]} \kappa(\theta)$ is achieved by $\theta$ that satisfies

$\frac{\mathrm{d} \kappa}{\mathrm{d} \theta}=0$

or

$2 C_{4} e^{2 i \theta}+C_{3} e^{i \theta}-C_{2} e^{-i \theta}-2 C_{1} e^{-2 i \theta}=0$

Let $z=e^{i \theta}$, then (98) becomes (48), which is a fourth order polynomial equation of $z$. There are four solutions of $z$ to this equation. However, only those solutions on the unit circle are potential candidates for $\kappa(\theta)$ to reach minimum. Because $\kappa(\theta)=p_{1}\left(e^{i \theta}\right)$, it is obvious that $\min _{\theta} \kappa(\theta)>0$ if and only if $p_{1}\left(z_{i}^{*}\right)>0$ for all the solutions $z_{i}^{*}$ of (48) that are on the unit circle.

It is easy to see that the second inequality of (96) is equivalent to $-2\left|g_{2}\right|>g_{1}$. This completes the proof.

Proof of Theorem 6. First, assume $a_{2} \neq 0, a_{3} \neq 0$, and $a_{23} \neq 0$. Note that $\min \eta(\alpha, \beta)$ is achieved by $(\alpha, \beta)$ that satisfy

$\frac{\partial \eta}{\partial \alpha}=0, \frac{\partial \eta}{\partial \beta}=0$

or

$a_{2} \sin (\alpha)+a_{23} \sin (\alpha+\beta)=0$,

$a_{3} \sin (\beta)+a_{23} \sin (\alpha+\beta)=0$.

It follows from (99) and (100) that (62) holds. By using (62), (99) becomes

$\sin (\alpha)\left[a_{2} a_{3}+a_{3} a_{23} \cos (\beta)+a_{2} a_{23} \cos (\alpha)\right]=0$.

It can be seen that a solution of (101) must satisfy either

$a_{2} a_{3}+a_{3} a_{23} \cos (\beta)+a_{2} a_{23} \cos (\alpha)=0$,

or

$\sin (\alpha)=0$

For (102), we have

$\cos (\beta)=-\frac{a_{2}}{a_{23}}-\frac{a_{2}}{a_{3}} \cos (\alpha)$.

From (62) and (104), we obtain

$1-\left[\frac{a_{2}}{a_{23}}+\frac{a_{2}}{a_{3}} \cos (\alpha)\right]^{2}=\frac{a_{2}^{2}}{a_{3}^{2}}\left[1-\cos ^{2}(\alpha)\right]$,

which can be solved for $\cos (\alpha)$ to obtain (59). A substitution of (104) by (59) yields (60). Because $|\cos (\alpha)| \leq 1$ and $|\cos (\beta)| \leq 1$, a real solution of (59) and (60) exists if and only if (57) and (58) hold. A substitution of (52) by (59)-(62) yields (63).

For (103), one has $\alpha=0$ or $\alpha=\pi$. For $\alpha=0$, we have

$$
\begin{aligned}
\min _{\alpha, \beta} \eta(\alpha, \beta) & =\min _{\beta}\left\{1+a_{2}+a_{3} \cos (\beta)+a_{23} \cos (\beta)\right\} \\
& =1+a_{2}-\left|a_{3}+a_{23}\right|,
\end{aligned}
$$

which is (54). For $\alpha=\pi$, we have

$$
\begin{aligned}
\min _{\alpha, \beta} \eta(\alpha, \beta) & =\min _{\beta}\left\{1-a_{2}+a_{3} \cos (\beta)-a_{23} \cos (\beta)\right\} \\
& =1-a_{2}-\left|a_{3}-a_{23}\right|
\end{aligned}
$$

which is (55).

Finally, if $a_{2}=0$, then obviously $\alpha$ and $\beta$ can be chosen such that

$\min _{\alpha, \beta} \eta(\alpha, \beta)=1-\left|a_{3}\right|-\left|a_{23}\right|$ 
This is already included in (54) and (55). It can be similarly shown that other cases $\left(a_{3}=0\right.$ or $\left.a_{23}=0\right)$ are also already included in (54) and (55). This completes the proof.

\section{References}

[1] Avellar C, and Hale J. On the zeros of exponential polynomials. Journal of Mathematical Analysis and Applications, vol. 73, no. 2, pp. 434-452, 1980.

[2] Corduneanu C. Almost Periodic Functions. Interscience Publishers, 1968.

[3] Doyle J. Analysis of feedback systems with structured uncertainties. IEE Proceedings D, vol. 129, no. 6, pp. 242250, 1982 .

[4] Doyle J, Wall J, and Stein G. Performance and robustness analysis for structured uncertainty. In Proceedings of the 21th IEEE Conference on Decision and Control, pp. 629-636, 1982.

[5] Gu K. A review of some subtleties of practical relevance for time-delay systems of neural type. ISRN Applied Mathematics, vol. 2012, article ID 725783, 46 pages, 2012.

[6] Gu K, and Zheng X. Stability crossing set for systems with three scalar delay channels. International Journal of Dynamics and Control, vol. 2, pp. 164-197, 2014.

[7] Hale J, and Verduyn Lunel S. Introduction to Functional Differential Equations. Springer, New York, NY, USA, 1993.

[8] Hale J, and Verduyn Lunel S. Strong stabilization of neutral functional differential equations. IMA Journal of Mathematical Control and Information, vol. 19, no. 1-2, pp. 5-23, 2002.

[9] Henry D. Linear autonomous neutral functional differential equations. Journal of Differential Equations, vol. 15, no. 1, pp. 106-128, 1974.

[10] Ma Q, Gu K, and Choubedar N. Further results on the strong stability of difference equations of continuous time. In Proceedings of the 20th World Congress of the International Federation of Automatic Control, Toulouse, France, July 914, pp. 13860-13865, 2017.

[11] Melvin W. Stability properties of functional difference equations. Journal of Mathematical Analysis and Applications, vol. 48, no. 3, pp. 749-763, 1974.

[12] Michiels W, Mondié S, Roose D, and Dambrine M. The effect of approximating distributed delay control laws on stability. In Advances in Time-Delay Systems, S.-I. Niculescu and K. Gu, Eds., pp. 207-222, Springer, Berlin, 2004.

[13] Mirkin L. On the approximation of distributed-delay control laws. Systems and Control Letters, vol. 51, no. 5, pp. 331-342, 2004.

[14] Mondié S, Dambrine M, and Santos O. Approximation of control laws with distributed delays: a necessary condition for stability. Kybernetika, vol. 38, no. 5, pp. 541-551, 2002.

[15] Naghnaeian M, and Gu K. Stability crossing set for systems with two scalar-delay channels. Automatica, vol. 49, no. 7, pp. 2098-2106, 2013.

[16] Packard A, and Doyle J. The complex structured singular value. Automatica, vol. 29, no. 1, pp. 71-109, 1993.

[17] Palmor Z. Stability properties of Smith dead-time compensator controller. International Journal of Control, vol. 32, no. 6, pp. 937-949, 1980.
[18] Pepe P. The Liapunov's second method for continuous time difference equations. International Journal of Robust and Nonlinear Control, vol. 13, no. 15, pp. 1389-1405, 2003.

[19] Shaikhet L. Lyapunov Functionals and Stability of Stochastic Difference Equations. Springer-Verlag, London, 2011.

[20] Zhong Q. On distributed delay in linear control laws-Part I: Discrete-delay implementations. IEEE Transactions on Automatic Control, vol. 49, no. 11, pp. 2074-2080, 2004.

[21] Zhou K, Doyle J, and Glover K. Robust and Optimal Control. Prentice Hall, Upper Saddle River, NJ, USA, 1996. 